\title{
Conus Medullaris Syndrome due to Ligamentum Flavum Thickening and Intervertebral Disc Bulging
}

\author{
Matthew Amodeo, $M D^{1}$, Leah Bowlin, DO $O^{1}$, Radhika Manne, MD ${ }^{1}$, Chloe Mccloskey, $M D^{1}$, Eric Canlas ${ }^{1}$, \\ Franklin E Caldera, $D O^{1}$ and Yejia Zhang $M D, P h D^{1,2^{*}}$
}

${ }^{1}$ Department of Physical Medicine \& Rehabilitation, Perelman School of Medicine, University of Pennsylvania, USA

${ }^{2}$ Translational Musculoskeletal Research Center (TMRC), Corporal Michael J. Crescenz Veterans Affairs Medical Center, USA

\begin{abstract}
A 72-year-old male with past medical history of common chronic illness presented to a rehabilitation clinic with a chief complaint of trouble walking and falls over 6 months. On detailed questioning, he has developed progressive bladder incontinence and unable to achieve erection. Physical exam revealed normal rectal tone and minimal bladder retention. Lumbar spine MRI shows severe spinal canal stenosis, with posterior elements impinging on the Conus Medullaris. He underwent T9-11 laminectomy. Upon follow up nearly 2-years post-operation, he is continent of bladder and able to walk with a rolling walker. The main differential diagnosis was Cauda Equina syndrome. Although both syndromes are surgical emergencies, accurate diagnosis would affect the surgical approach and outcome.
\end{abstract}

\section{Keywords}

Conus medullaris syndrome, Cauda equina syndrome

\section{History}

A 72-year-old male with past medical history of hypertension, hyperlipidemia, chronic obstructive pulmonary disease, and depression. He also had chronic low back pain radiating to both legs for over 10-years and was managed with methadone. He presented to the wheelchair clinic in November 2018 , because of difficulty in walking due to bilateral leg weakness over 6-months, which had worsened progressively for several weeks. He used a walker initially, but as his symptoms had worsened, together with multiple falls, he presented to request a scooter. On detailed questioning, he reported 2-3 weeks of progressive bladder incontinence; he could not "hold it in" long enough to reach the bathroom. During the same period as the bladder incontinence, he also had constipation, and was unable to achieve an erection. He was recommended to go to the Emergency Department for further evaluation.

\section{Physical Examination}

The patient was 5 ' 8 , with a weight of $150 \mathrm{lbs}$. At the Emergency Department, he was found to have weakness of hip flexors and extensors. He had normal rectal tone, voided 400 $\mathrm{ml}$ of urine on his own and had a residual volume of $42 \mathrm{ml}$ as measured by ultrasound bladder scan. He had intact perianal sensation, and "normal" patellar tendon reflex.

\section{Imaging Studies}

An MRI scan of the lumbar spine reported various diffuse disc bulges and ligamentum flavum thickening at L2-5 levels, with severe bilateral L5 neuroforaminal narrowing. It was fortunate that the lumbar spine MRI showed the thoracic (T) 10-T12 levels as well. On this image (Figure 1), the patient's spinal cord begins to taper at the T11 level and ends at the L1 level. On the T2 weighted image, there was a severe canal stenosis, with severe compression of the spinal cord (Figure $1 \mathrm{~A}$, yellow arrow). On the T1 weighted image, posterior elements, including the ligamentum flavum (Figure 1B, yellow arrow) were visible, with the adjacent spinal cord compressed to a thin layer (red arrow).

*Corresponding author: Yejia Zhang, MD, PhD, Department of Physical Medicine \& Rehabilitation, Translational Musculoskeletal Research Center Perelman School of Medicine, University of Pennsylvania, USA

Accepted: December 26, 2020

Published online: December 28, 2020

Citation: Amodeo M, Bowlin L, Manne R, et al. (2020) Conus Medullaris Syndrome due to Ligamentum Flavum Thickening and Intervertebral Disc Bulging. J Orthop Rheumatism 4(1):7880

Copyright: (c) 2020 Amodeo M, et al. This is an open-access article distributed under the terms of the Creative Commons Attribution License, which permits unrestricted use, distribution, and reproduction in any medium, provided the original author and source are credited. 



Figure 1: Severe canal stenosis at T10-11 level with spinal cord signal abnormality extending superiorly from T11 to the mid T10 vertebral body level. (A) T2-weighted mid-sagittal image of lumbar spine MRI included T11 and partial T10 vertebral bodies; (B) T1weighted image; Yellow arrows- Hypertrophic Posterior Elements Including Ligamentum Flavum; Red Arrow- Compressed Spinal Cord.

An additional MRI of the thoracic spine report stated that at T10-T11, there is severe canal stenosis with compressive myelopathy, with cord signal abnormality extending superiorly to the mid T10 vertebral body level. This is due primarily to severe ligamentum flavum thickening, which may be partially calcified, with a small anterior disc osteophyte complex. A neurosurgeon was consulted.

\section{Surgery and Post-Operative Course}

T9-11 laminectomy was performed by a neurosurgeon 7 days after the patient's initial clinical visit. Specifically, the lower portion of the T9 lamina, complete T10 lamina, and the superior portion of the T11 lamina were removed.

Post-operatively, the patient reported persistent pain in his back at the incision site, but the radiating leg pain had resolved. His urinary incontinence had resolved as well. The patient had an uncomplicated post-operative course and completed a dexamethasone taper for pain control.

The patient was discharged after an inpatient stay with Physical Medicine and Rehabilitation. Upon telephone follow-up in September of 2020, the patient was continent of bladder and bowel, and was able to walk with a rolling walker.

\section{Differential Diagnosis and Clinical Decision- Making}

This patient was a surgical emergency because he lost bladder control and erectile function over a period of a few weeks. The main differential diagnosis includes conus medullaris compression and Cauda Equina syndrome. The decision about the level at which to operate depends on the differential diagnosis.

The Conus Medullaris is the distal end of the spinal cord, which tapers towards the lumbar region. This tapering occurs at around the L1 vertebra but can vary from as high as the T11 (thoracic) or as low as the L3 vertebrae. The Cauda Equina are nerve roots that stem from the Conus Medullaris in the region between the lumbar and sacral sections of the spine. The Conus Medullaris is the caudal end of the spinal cord, which is part of the central nervous system. The Cauda Equina (from Latin horse's tail) is a bundle of spinal nerves and spinal nerve rootlets, consisting of the second through fifth lumbar nerve pairs, the first through fifth sacral nerve pairs, and the coccygeal nerve, all of which arise from the lumbar enlargement and the Conus Medullaris of the spinal cord. Although the Cauda Equina resides within the spinal canal, it is part of the peripheral nervous system.

Unlike Cauda Equina syndrome, which will only have lower motor neuron deficits, Conus Medullaris syndrome can lead to a combination of upper and lower motor neuron features. A comparison of the two syndromes and our patient's presentation is summarized in Table 1.

\section{Discussion}

This patient's symptoms and signs were most consistent 
Citation: Amodeo M, Bowlin L, Manne R, et al. (2020) Conus Medullaris Syndrome due to Ligamentum Flavum Thickening and Intervertebral Disc Bulging. J Orthop Rheumatism 4(1):78-80

Table 1: Comparison between Conus Medullaris and Cauda Equina Syndromes.

\begin{tabular}{|l|l|l|}
\hline Conus Medullaris Syndrome & Cauda Equina Syndrome & The Patient's Presentation \\
\hline Spastic Bladder & Flaccid Bladder & $\begin{array}{l}\text { Able to Avoid with Low Residual Volume, Unable } \\
\text { To "Make It to The Bathroom", Consistent with } \\
\text { Spastic Bladder. }\end{array}$ \\
\hline Rectal Tone Preserved & Decreased or Loss of Rectal Tone & Normal Rectal Tone \\
\hline Bulbocavernosus Reflex Pre-Served & Bulbocavernosus Reflex Ab-Sent & Not Tested \\
\hline Saddle Anesthesia & Saddle Anesthesia & Had Perianal Sensation. \\
\hline Symmetric Presentation & Asymmetric Presentation & Symmetric Presentation \\
\hline Mild Muscle Weakness & Severe Muscle Weakness & Somewhat Severe Muscle Weak-Ness \\
\hline Preserved Deep Tendon Reflex & Absent or Decreased Deep Tendon Reflex & "Normal" \\
\hline
\end{tabular}

with Conus Medullaris syndrome, although he had some lumbar radiculopathy that caused leg muscle weakness, resulting in falls. He had degenerative lumbar spine changes which could confound the diagnosis. A clearer description of the patellar tendon reflex would also have been helpful. In our patient, the normal rectal tone and spastic bladder were the crucial findings in confirming the diagnosis and identifying the correct site for operative intervention.

Both syndromes are rare [1].One study estimated that there could be 1016 new causes of cauda equina syndrome and 449 new cases of conus medullaris syndrome per year in the U.S. [2] Conus medullaris syndrome often occurs posttraumatically [3], or as a result of spinal column tumors. It is rarely encountered in the setting of degenerative spinal stenosis. Although the value of timing of surgical intervention in the setting of incomplete spinal cord injury is unclear at this time [4,5], prompt MR imaging and surgery resulted in restored function for our patient.

\section{Acknowledgement}

The authors gratefully thank Martin F. Heyworth, MD, for critically editing the manuscript.

\section{References}

1. Rider LS, Marra EM (2020) Cauda equina and conus medullaris syndromes. In: StatPearls Treasure Island (FL) StatPearls Publishing LLC.

2. Podnar S (2007) Epidemiology of cauda equina and conus medullaris lesions. Muscle Nerve 35: 529-531.

3. Taylor TK, Coolican MJ (1988) Injuries of the conus medullaris. Paraplegia 26: 393-400.

4. Radcliff KE, Kepler CK, Delasotta LA, et al. (2011) Current management review of thoracolumbar cord syndromes. Spine J 11: 884-892.

5. Harrop JS, Hunt GE Jr, Vaccaro AR (2004) Conus Medullaris and Cauda Equina Syndrome as a result of Traumatic Injuries: Management principles. Neurosurg Focus 16: e4.

DOI: $10.36959 / 479 / 436$

Copyright: (c) 2020 Amodeo M, et al. This is an open-access article distributed under the terms of the Creative Commons Attribution License, which permits unrestricted use, distribution, and reproduction in any medium, provided the original author and source are credited. 